\title{
Invasive mycoses in patients with connective tissue disease from Southern China: clinical features and associated factors
}

Minxi Lao ${ }^{1+}$, Zhongping Zhan ${ }^{2 \dagger}$, Fan $\mathrm{Su}^{1+}$, Hao $\mathrm{Li}^{2}$, Zheng Yang ${ }^{3}$, Haihong Chen ${ }^{4}$, Liuqin Liang ${ }^{2^{*}}$ and Dongying Chen $^{2^{*}}$

\begin{abstract}
Background: A retrospective study was performed to investigate the clinical features and associated factors of invasive mycoses (IM) in patients with connective tissue disease (CTD) from Southern China.

Methods: Demographic and clinical data were recorded. Associated factors were analyzed by logistic regression analysis.

Results: A total of 6911 patients with CTD were included. IM was diagnosed in 32 patients (incidence, 0.5\%). IM was predominant in patients with ANCA-associated vasculitis (AAV) (incidence, 1.5\%, 7/480). Lung was commonly involved (30/ 32, 93.8\%). Aspergillus spp. (81.3\%) were the leading strain. The positive rate of fungi detection in sputum culture was 69.0\%. Serum galactomannan (GM) test was positive in bronchoalveolar lavage fluid (BALF) from seven (7/10, 70.0\%) patients. Ten patients died (31.3\%), including three with AAV (42.9\%) and seven with SLE (36.8\%). Penicillium marneffei was the most fatal (mortality, 100\%). Non-survivors had higher prevalence of leukopenia (30.0\% vs $4.5 \%, P=0.04$ ), lymphopenia (100.0\% vs $59.1 \%, P=0.02)$, elevated serum creatinine $(70.0 \%$ vs $27.3 \%, P=0.02)$, and co-infection $(70.0 \%$ vs $18.2 \%, P=0.004)$ than survivors. Multivariate logistic regression analysis showed that lymphopenia [odds ratio $(\mathrm{OR})=3.28,95 \%$ confidence interval (Cl) 1.29-8.38, $P=0.01]$ and median-to-high dose of glucocorticoid (GC) $[\mathrm{OR}=3.40,95 \% \mathrm{Cl} 1.04-11.13, P=0.04]$ were associated with IM in patients with CTD.
\end{abstract}

Conclusions: IM tended to develop in patients with AAV, resulting in high mortality. Sputum culture and GM test in BALF were effective methods to distinguish IM. Vigilance against lymphopenia, impaired kidney function, and co-infection improved the prognosis of IM.

Keywords: Connective tissue disease, Invasive mycosis, Lymphopenia, Glucocorticoid, Co-infection

\section{Background}

Connective tissue disease (CTD) is a group of autoimmune diseases caused by immune system disorder. Despite considerable progress in immunosuppressive and supportive treatment, infection is still one of the major threats to patients with CTD [1]. A multicenter retrospective study found that $65.8 \%$ of the deaths were imputable to infection in patients with systemic lupus

\footnotetext{
* Correspondence: lliuq@mail.sysu.edu.cn; cdongying@163.com

${ }^{\dagger}$ Minxi Lao, Zhongping Zhan and Fan Su contributed equally to this work.

${ }^{2}$ Department of Rheumatology, The First Affiliated Hospital of Sun Yat-sen

University, 58 Zhongshan 2nd Road, Guangzhou 510080, China

Full list of author information is available at the end of the article
}

erythematosus (SLE) [2]. During the induction therapy of ANCA-associated vasculitis (AAV), the incidence of severe infection was estimated to be $34.7 \%$ [3]. Infection was also reported to be the leading cause of non-systemic sclerosis (SSc)-related deaths (18.2\%) [4]. Opportunistic fungal infection was an independent factor with mortality in polymyositis/dermatomyositis (PM/DM) patients [5].

Invasive fungal disease (IFD) was well studied in patients with AIDS and organ transplant recipients [6, 7]. A few studies illustrated that patients with CTD were also predisposed to IFD. A systemic review included 182 studies reported that the prevalence of IFD in lupus patients ranged between 0.6 and $3.2 \%$ [8]. A total of 461

(c) The Author(s). 2019 Open Access This article is distributed under the terms of the Creative Commons Attribution 4.0 International License (http://creativecommons.org/licenses/by/4.0/), which permits unrestricted use, distribution, and 
cases of IFD were included in the review, and mold infection accounted for $21.9 \%$. Death rate increased fourto sixfold in lupus patients with IFD than those without $[9,10]$. Another research showed that among 87 episodes of severe infection in patients with new diagnosis of AAV, 3 (3.4\%) were caused by Aspergillus spp. [3]. A Chinese research exploring invasive pulmonary aspergillosis (IPA) in hospitalized patients with AAV reported that mortality of aspergillosis was as high as $57.2 \%$ [11].

Although IFD, especially invasive mycosis (IM) caused by molds, is a challenging cause of morbidity and mortality of CTD, our present understanding is mostly limited to the typical clinical manifestation and radiological presentation. Few studies were designed to focus on IM in patients with CTD. In fact, the great variations and protean manifestation of these clinical features often lead to misdiagnosis [12]. The timing of treatment initiation against IM is not fully determined.

In this study, we performed a retrospective study in patients with SLE, AAV, PM/DM, rheumatoid arthritis (RA), and SSc, aiming to identify the epidemiology and the associated factors affecting the development of IM infected by molds in patients with CTD from Southern China.

\section{Methods}

\section{Study design and case definition}

We performed a retrospective study on the inpatients from the First Affiliated Hospital of Sun Yat-Sen University from January 1, 2007, to December 31, 2017. The ICD-10 coding of discharged diagnoses was used to identify patients with CTD and IM (relevant ICD-10 coding in Appendix). Records were re-evaluated by a rheumatologist (Minxi Lao) and a respirologist (Haihong Chen). SLE was diagnosed according to the 1997 American College of Rheumatology classification criteria [13]. RA was diagnosed according to the 1987 ACR revised criteria for the classification of rheumatoid arthritis [14]. AAV was determined using the definitions of the Chapel Hill nomenclature $[15,16]$. PM/DM was established referring to the classification criteria proposed by Bohan [17]. The diagnosis of SSc was conducted based on 1980 ARA classification criteria for systemic sclerosis [18]. IM infected by molds was determined using the EORTC/ MSG 2008 Guidelines [19] and classified into three categories namely proven, probable, and possible. Diagnosis of proven IM required culture or microscopic evidence of the molds from a normally sterile site. A probable case required the presence of a host factor, mycological evidence \{identification of mold species in respiratory samples [positive twice in microscopic examination and culture of sputum, or positive once in bronchoalveolar lavage fluid (BALF)], or identification of fungal antigen in blood [twice elevated serum 1,3- $\beta$-d-glucan level (G test)], or positive twice for serum galactomannan (GM) antigen)\}, and clinical manifestation as well as radiographic imaging specific to the disease entity. Possible IM was a case with a host factor and clinical presentation, but no mycological evidence. In the interest of capturing cases which were most likely to be truly IM, only proven and probable cases were included in this study. Superficial infection, namely cutaneous and mucosal infection, was excluded. Patients with incomplete data were excluded. Co-infection with other agents including bacteria, virus, or superficial fungi was diagnosed according to the criteria described previously [20]. The outcome of IM was classified into two categories including improvement and progression in our research. Improvement was defined as both clinical symptoms and radiographic imaging partially resolved. Progressive disease was defined as clinical symptoms and radiographic imaging worsened. Inpatients with CTD but no history of any infection from the same period of time were chosen as controls. Cases and controls were matched 1:2 by age, gender, subtype, and the duration of CTD (from CTD onset to IM).

\section{Clinical variables}

Demographic and clinical data were collected from medical records. Clinical characteristics of IM included symptoms and sign, sites of involvement, and medication history. CTD-associated pneumonia was established according to the Chinese expert-based consensus statement [21]. Laboratory data included routine blood tests, erythrocyte sedimentation rate (ESR), and serum C-reactive protein (CRP). Specimen culture and biopsy finding were recorded if available. Medication history included daily dose of glucocorticoid (GC) and the use of immunosuppressive agents within the 3 months prior to IM. Median-to-high dose of GC was defined as the average dose of equivalent prednisone over $0.5 \mathrm{mg} / \mathrm{kg} / \mathrm{day}$ within the previous 3 months. The dose of GC was converted using the following equation: $1 \mathrm{mg}$ of prednisone $=0.8 \mathrm{mg}$ of methylprednisolone $=0.15 \mathrm{mg}$ of dexamethasone.

\section{Statistical analysis}

Statistical analysis was computed with SPSS 19.0 software (SPSS Inc., Chicago, IL, USA). The descriptive variables included mean (S.D.) or median (interquartile range, IQR) depending on the distribution of continuous variables and number (percentage) for qualitative variables. Between-group comparison was evaluated with chi-square test or Fisher exact test for categorical variables and Student's $t$ test for continuous variables with normal distribution. Between-group comparison was evaluated using the Mann-Whitney $U$ test for continuous variables with non-normal distribution. Variables with $P$ value $<0.10$ from the univariate analysis were considered as candidates for the multivariate analysis. The multivariate analysis was preceded by a forward procedure to identify the factors associated with IM. 
Associated factors were reported with $P$ values, odds ratio (OR), and $95 \%$ confidence interval (CI). $P$ value $<0.05$ was considered statistically significant.

\section{Results}

\section{Demographic data}

A total of 6911 inpatients with CTD (including 4196 patients with SLE, 728 with PM/DM, 666 with primary Sjogren's syndrome (pSS), 530 with vasculitis, 407 with RA, 197 with SSc, 102 with spondyloarthrosis (SpA), and 85 with mixed connective tissue disease (MCTD)) were selected. IM was diagnosed in 54 patients including 29 patients with SLE, 15 patients with AAV, 5 patients with PM/DM, 4 patients with RA, and 1 patient with SSc. Sixteen patients were excluded for possible diagnosis of IM, and six patients were excluded for data incompleteness. Finally, 32 patients (included 19 patients with SLE, 7 with AAV, 3 with PM/DM, 2 with RA, and 1 with SSc) were included in our research. Female-to-male ratio was 25:7. The incidence of IM was $0.5 \%(32 / 6911)$. To be specific, the incidence was $1.5 \%(7 / 480)$ in patients with AAV, 0.5\% (19/4196) in SLE, 0.5\% (2/407) in RA, 0.5\%
$(1 / 197)$ in SSc, and 0.4\% (3/728) in PM/DM. Proven infection accounted for $18.8 \%(6 / 32)$ of the cases, and probable infection for $81.3 \%(26 / 32)$. All patients were of Chinese Han ethnicity. Of the 32 patients enrolled, mean age was $42.4 \pm 15.8$ years old (range, 14-68). Characteristics of patients with CTD are shown in Table 1. Kidney involvement occurred in 15 (78.9\%) patients with SLE and in 4 (57.1\%) patients with AAV. CTD-associated interstitial pneumonia was predominant in patients with AAV (42.9\%), followed by PM (33.3\%) and SLE (5.3\%). During the 3 months prior to IM, 22 (68.8\%) patients received GC treatment, among which $50.0 \%$ (11/ 22) received median-to-high dose of GC. Cyclophosphamide (CYC), mycophenolate mofetil (MMF), cyclosporine A (CsA), and methotrexate (MTX) were prescribed to 9 (28.1\%), 3 (9.4\%), 3 (9.4\%), and 2 (6.3\%) patients, respectively. No patients received biologic treatment.

\section{Characteristics and laboratory data of IM in patients with CTD}

The lung (30/32, 93.8\%) was the most commonly involved. Two patients were diagnosed with fungal

Table 1 Characteristics of patients diagnosed with CTD and IM

\begin{tabular}{|c|c|c|c|c|c|}
\hline Characteristics & $\operatorname{SLE}(n=19)$ & $\operatorname{AAV}(n=7)$ & PM/DM $(n=3)$ & RA $(n=2)$ & $\operatorname{SSC}(n=1)$ \\
\hline \multicolumn{6}{|l|}{ Demographic characteristics } \\
\hline Sex, male to female & $5: 14$ & $1: 6$ & $1: 2$ & $0: 2$ & $0: 1$ \\
\hline Age, years, mean $\pm S D$ & $35.6 \pm 15.3$ & $53.1 \pm 13.0$ & $57.0 \pm 4.4$ & $46.5 \pm 9.2$ & 46.0 \\
\hline Duration of CTD, months, median (IQR) & $4(1.0,21.0)$ & $3(0.3,84.0)$ & $120(61.5,180.0)$ & 204(186.0,222.0) & 108.0 \\
\hline Diabetes mellitus, $n$ (\%) & $3(15.8)$ & $1(14.3)$ & $0(0)$ & $0(0)$ & $0(0)$ \\
\hline Nephritis, $n$ (\%) & 15(78.9) & $4(57.1)$ & $0(0)$ & $0(0)$ & $0(0)$ \\
\hline Interstitial pneumonia, n (\%) & $1(5.3)$ & $3(42.9)$ & $1(33.3)$ & $0(0)$ & $1(100.0)$ \\
\hline \multicolumn{6}{|l|}{ Laboratory data } \\
\hline WBC count $(\times 109)$, mean \pm SD & $9.5 \pm 6.7$ & $10.7 \pm 6.0$ & $12.9 \pm 5.6$ & $8.8 \pm 1.2$ & 6.6 \\
\hline Lymphocyte count $(\times 109)$, mean \pm SD & $0.9 \pm 1.0$ & $0.8 \pm 0.7$ & $1.1 \pm 0.4$ & $1.5 \pm 1.0$ & 0.7 \\
\hline Hemoglobin $(g / l)$, mean $\pm S D$ & $96.1 \pm 26.2$ & $87.3 \pm 27.5$ & $116.7 \pm 2.5$ & $114.5 \pm 3.5$ & 109.0 \\
\hline Serum albumin $(\mathrm{g} / \mathrm{l})$, mean $\pm \mathrm{SD}$ & $28.1 \pm 7.4$ & $32.8 \pm 3.5$ & $27.0 \pm 11.3$ & $35.5 \pm 4.9$ & 32.0 \\
\hline Elevated serum creatinine, $n(\%)$ & $6(31.6)$ & $6(85.7)$ & $0(0)$ & $0(0)$ & $0(0)$ \\
\hline $\operatorname{lgM}(\mathrm{g} / \mathrm{l})$, median (IQR) & $0.7(0.3,0.9)$ & $0.5(0.4,0.8)$ & $1.2(1.1,1.4)$ & $0.7(0,0.5)$ & 0.3 \\
\hline $\operatorname{lgG}(g / l)$, median (IQR) & 15.4(7.4,17.4) & $13.4(12.6,20.4)$ & $15.2(14.4,16.0)$ & $14.8(0,11.8)$ & 14.1 \\
\hline ESR (mm/h),median (IQR) & $45.0(39,58.5)$ & $63.0(60,66.0)$ & $87.5(81.8,93.3)$ & $49.5(43.3,55.8)$ & 30.0 \\
\hline CRP (mg/l), median (IQR) & $11.0(6.2,33.4)$ & $12.3(6.3,20.1)$ & $102.0(52.7,139.0)$ & $65.6(34.4,96.8)$ & 0.6 \\
\hline \multicolumn{6}{|l|}{ Treatment prior to IM } \\
\hline Median-to-high dose of GC, $n$ (\%) & $6(31.6)$ & $3(42.9)$ & $2(66.7)$ & $0(0)$ & $0(0)$ \\
\hline CYC, $n(\%)$ & $4(21.1)$ & $4(57.1)$ & $1(33.3)$ & $0(0)$ & $0(0)$ \\
\hline MMF, n (\%) & $3(15.8)$ & $0(0)$ & $0(0)$ & $0(0)$ & $0(0)$ \\
\hline CsA, n (\%) & $2(10.5)$ & $0(0)$ & $0(0)$ & 1(50.0) & $0(0)$ \\
\hline MTX, n (\%) & $1(5.3)$ & $0(0)$ & $0(0)$ & $1(50.0)$ & $0(0)$ \\
\hline
\end{tabular}

AAV ANCA-associated vasculitis, CRP C-reactive protein, CSA cyclosporine A, CTD connective tissue disease, CYC cyclophosphamide, ESR erythrocyte sedimentation rate, GC glucocorticoid, IM invasive mycoses, IQR interquartile range, MMF mycophenolate mofetil, MTX methotrexate, $P M / D M$ polymyositis/dermatomyositis, $R A$ rheumatoid arthritis, SD standard deviation, SLE systemic lupus erythematosus, SSC systemic sclerosis, WBC white blood cell 
sinusitis (2/32, 6.3\%) (Additional file 1: Figure S1 A-D). Pulmonary symptoms are presented in Table 2. Aspergillus spp. were the leading pathogens, causing 26 (81.3\%) cases of infection. Mucor spp. and Penicillium spp. caused $3(9.4 \%)$ and $2(6.3 \%)$ infections, respectively. One (3.1\%) patient was infected with both Aspergillus spp. and Mucor spp. Most of the causative fungi were identified in sputum culture $(20 / 29,69.0 \%)$, while a small proportion was found in BALF $(3 / 10,30.0 \%)$. Aspergillus fumigates was common $(n=10)$. Other causative agents included Penicillium marneffei $(n=2)$, Mucor $(n=2)$, Aspergillus flavus $(n=2)$, Aspergillus niger $(n=1)$, and Rhizopus $(n=1)$. Co-infection with mix fungi was found in $4(12.5 \%)$ lupus patients and 1 (3.1\%) AAV patient. Serum G test was performed in 23 patients and positive in 11 (47.8\%). GM test was performed in 26 patients and positive in 9 (34.6\%). The positive rate of the GM test was high in BALF (7/10, $70.0 \%)$. Six patients were diagnosed on the basis of biopsy, including four by bronchofiberscopy and two by nasal endoscopy (Additional file 1: Figure S1 G, H).

\section{Pulmonary manifestation of IM in patients with CTD} Radiographic manifestation from pulmonary computed tomography (CT) scan is shown in Table 2. (Additional file 1: Figure S1E, F). Most of the nodules (85.7\%) were small

Table 2 Pulmonary manifestation of IM in patients with CTD

\begin{tabular}{|c|c|}
\hline & Total $(n=30)$ \\
\hline \multicolumn{2}{|l|}{ Clinical symptoms } \\
\hline Fever, $n(\%)$ & $20(66.7)$ \\
\hline Cough, $n(\%)$ & $24(80.0)$ \\
\hline Sputum, $n(\%)$ & $21(70.0)$ \\
\hline Dyspnea, $n(\%)$ & $8(26.7)$ \\
\hline Hemoptysis, n (\%) & $6(20.0)$ \\
\hline Chest distress, $n(\%)$ & $3(10.0)$ \\
\hline Chest pain, $n(\%)$ & $2(6.7)$ \\
\hline \multicolumn{2}{|l|}{ Radiographic manifestation } \\
\hline Nodules, $n(\%)$ & $14(46.7)$ \\
\hline$<3 \mathrm{~cm}$ in diameter, $n(\%)$ & 12(85.7) \\
\hline$>3 \mathrm{~cm}$ in diameter, $n(\%)$ & $2(14.3)$ \\
\hline Single nodule, $n(\%)$ & $6(42.9)$ \\
\hline Multiple nodules, $n$ (\%) & $8(57.1)$ \\
\hline Cavitary lesions, $n(\%)$ & $11(36.7)$ \\
\hline Ground glass opacity, n (\%) & $8(26.7)$ \\
\hline Halo sign, $n(\%)$ & $3(10.0)$ \\
\hline \multicolumn{2}{|l|}{ Location } \\
\hline Unilateral involvement, $n$ (\%) & $13(43.3)$ \\
\hline Bilateral involvement, $n(\%)$ & $17(56.7)$ \\
\hline
\end{tabular}

CTD connective tissue disease, IM invasive mycoses nodules with a diameter less than $3 \mathrm{~cm}$. Nodules with blurred edges were found in $42.9 \%$ of the cases. A halo sign was reported in $3(10.0 \%)$ cases. Nearly $28.6 \%$ of the nodules were located adjacent to the pleura. Bilateral involvement was common (56.7\%). Patients with AAV had the highest rate of bilateral involvement (85.7\%), followed by SLE (52.6\%).

\section{Co-infection with other agents}

Broad-spectrum antibiotics were given to $17(53.1 \%)$ patients prior to IM diagnosis. Co-infection occurred in 11 (34.3\%) patients, 10 of which were confirmed with etiological evidence. Co-infection occurred in 8 (42.1\%) patients with SLE and $3(42.9 \%)$ patients with AAV. Nearly $54.5 \%(6 / 11)$ of the co-infection occurred in the lung. The other five cases of infection involved multiple organs. Bacteria-associated infection accounted for $63.6 \%$ (7/11) of the cases. Tuberculosis occurred in 1 (9.1\%) patient. Herpes zoster was diagnosed in 1 (9.1\%) patient clinically. Co-infection with two pathogens occurred in 3 (27.3\%) patients, 2 in SLE and 1 in AAV. Distributions of the infective sites and causal agents are shown in Additional file 2: Table S1.

\section{Treatment and outcomes}

More than half $(17 / 26,65.4 \%)$ of the patients infected with Aspergillus spp. were treated with voriconazole. Other treatments included amphotericin B $(3 / 26,11.5 \%)$, caspofungin $(2 / 26,7.7 \%)$, itraconazole $(2 / 26,7.7 \%)$, and micafungin $(1 / 26,3.8 \%)$. Amphotericin $B$ in combination with flucytosine was prescribed to four patients with mucormycosis. Patients infected with $P$. marneffei were given either caspofungin $(1 / 2,50.0 \%)$ or amphotericin $B$ $(1 / 2,50.0 \%)$. Operation was performed in two patients diagnosed with fungal sinusitis and one patient with aspergilloma. Follow-up data was obtained from 29 patients and lost in 3 . The median duration of follow-up was 9 months (IQR, 2.5, 15.0). Disease improved in 19 patients after anti-fungal treatment. Five patients (15.6\%) were admitted to the intensive care unit (ICU). Ten patients died (10/32, 31.3\%). Mortality was the highest in patients with AAV (3/7, 42.9\%), followed by SLE (7/19, 36.8\%). Infection with P. marneffei was the most fatal (mortality, 100\%). Aspergillosis (mortality, 26.9\%) and mucormycosis (mortality, 25.0\%) ranked the second and the third. Among deceased patients with aspergillosis, 5 (71.4\%) were infected with A. fumigates. Infection-induced respiratory failure was the primary cause $(10 / 10,100 \%)$. All the deaths occurred in patients with pulmonary IM $(10 / 30,33.3 \%)$. A comparison was performed between the survivors and non-survivors (Table 3). The incidence of leukopenia $(4.5 \%$ vs $30.0 \%$, $P=0.04)$ and lymphopenia $(59.1 \%$ vs $100.0 \%, P=0.02)$ was lower in the survivors. Elevated serum creatinine 
Table 3 Comparison between survivors and non-survivors with IM

\begin{tabular}{|c|c|c|c|}
\hline Characteristics & Survivor $(n=22)$ & $\begin{array}{l}\text { Non-survivor } \\
(n=10)\end{array}$ & $P$ value \\
\hline \multicolumn{4}{|l|}{ Demographic characteristics } \\
\hline Sex, male to female & $4: 18$ & $3: 7$ & 0.45 \\
\hline Age, years, mean $\pm S D$ & $41.6 \pm 15.6$ & $44.2 \pm 16.8$ & 0.68 \\
\hline Diabetes mellitus, $n(\%)$ & $2(9.1)$ & $2(20.0)$ & 0.39 \\
\hline $\begin{array}{l}\text { Interstitial pneumonia, } \\
n(\%)\end{array}$ & $4(18.2)$ & $2(20.0)$ & 0.90 \\
\hline Nephritis, n (\%) & $12(54.5)$ & $7(70.0)$ & 0.41 \\
\hline \multicolumn{4}{|l|}{ Laboratory data } \\
\hline Leukopenia, $n(\%)$ & $1(4.5)$ & $3(30.0)$ & $0.04^{*}$ \\
\hline Lymphopenia, n (\%) & 13(59.1) & $10(100)$ & $0.02^{*}$ \\
\hline Anemia, $n(\%)$ & $12(54.5)$ & $8(80.0)$ & 0.17 \\
\hline Hypoalbuminemia, $n$ (\%) & 14(63.6) & $9(90.0)$ & 0.12 \\
\hline $\begin{array}{l}\text { Elevated serum creatinine, } \\
n(\%)\end{array}$ & $6(27.3)$ & $7(70.0)$ & $0.02^{*}$ \\
\hline Co-infection, $n(\%)$ & $4(18.2)$ & $7(70.0)$ & $0.004^{*}$ \\
\hline $\begin{array}{l}\text { Co-infection with multiple } \\
\text { agents, } n(\%)\end{array}$ & $1(4.5)$ & $2(20.0)$ & 0.22 \\
\hline $\begin{array}{l}\text { Co-infection in multiple } \\
\text { sites, } n(\%)\end{array}$ & $0(0)$ & $5(50.0)$ & $<0.001^{*}$ \\
\hline \multicolumn{4}{|l|}{ Treatments prior to IM } \\
\hline $\begin{array}{l}\text { Median-to-high dose of } \\
\text { GC, } n(\%)\end{array}$ & $7(31.8)$ & $4(40.0)$ & 0.65 \\
\hline CYC, $n(\%)$ & $4(18.2)$ & $5(50.0)$ & 0.06 \\
\hline MMF, $n(\%)$ & $1(4.5)$ & $2(20.0)$ & 0.16 \\
\hline
\end{tabular}

CYC cyclophosphamide, GC glucocorticoid, IM invasive mycoses, MMF mycophenolate mofetil, SD standard deviation "Significant

(27.3\% vs $70.0 \%, P=0.02)$ and co-infection $(18.2 \%$ vs $70.0 \%, P=0.004$ ) were more frequent in non-survivors than survivors.

\section{Comparison between patients with or without IM}

A total of 64 patients were selected as controls, including 38 patients with SLE, 14 patients with AAV, 6 patients with PM/DM, 4 patients with RA, and 2 patients with SSc. A comparison of the demographic and clinical characteristics is shown in Table 4. The prevalence of CTD-associated interstitial pneumonia (18.8\% vs $3.1 \%$, $P=0.01)$ and lymphopenia $(71.9 \%$ vs $42.2 \%, P=0.01)$ was more predominant in the mycoses group than in the control group. ESR $(56 \mathrm{~mm} / \mathrm{h}$ vs $27 \mathrm{~mm} / \mathrm{h}, P=0.01)$ and CRP levels $(11.7 \mathrm{mg} / \mathrm{l}$ vs $2.9 \mathrm{mg} / \mathrm{l}, P=0.002)$ were higher in patients with IM than in those without. A higher proportion of patients received median-to-high dose of GC $(34.4 \%$ vs $9.4 \%, P<0.001)$ in the mycoses group than those in the control group.

Considering elevated ESR and CRP levels were nonspecific to IM, only interstitial pneumonia, lymphopenia,
Table 4 Comparison between patients with or without IM

\begin{tabular}{|c|c|c|c|}
\hline Characteristics & Case $(n=32)$ & $\begin{array}{l}\text { Control } \\
(n=64)\end{array}$ & $P$ value \\
\hline \multicolumn{4}{|l|}{ Demographic characteristics } \\
\hline Sex, male to female & $7: 25$ & $15: 49$ & 0.86 \\
\hline Age, years, mean $\pm S D$ & $42.4 \pm 15.8$ & $45.5 \pm 17.5$ & 0.44 \\
\hline $\begin{array}{l}\text { Duration of CTD, months, } \\
\text { median (IQR) }\end{array}$ & $6.0(1.0,75.0)$ & $24.0(7.5,87.0)$ & 0.19 \\
\hline Diabetes mellitus, $n(\%)$ & $4(12.5)$ & $3(4.7)$ & 0.17 \\
\hline Interstitial pneumonia, $n$ (\%) & 6(18.8) & $2(3.1)$ & $0.01^{*}$ \\
\hline Nephritis, $n$ (\%) & 19(59.4) & $29(45.3)$ & 0.19 \\
\hline \multicolumn{4}{|l|}{ Laboratory data } \\
\hline Leukopenia, $n(\%)$ & $4(12.5)$ & $11(17.2)$ & 0.55 \\
\hline Lymphopenia, n (\%) & 23(71.9) & $27(42.2)$ & $0.01^{*}$ \\
\hline Anemia, $n(\%)$ & $20(62.5)$ & $38(59.4)$ & 0.77 \\
\hline Hypoalbuminemia, n (\%) & 23(71.9) & $42(75.0)$ & 0.75 \\
\hline $\begin{array}{l}\text { Elevated serum creatinine, } \\
n(\%)\end{array}$ & 13(40.6) & $18(28.1)$ & 0.22 \\
\hline IgM (g/l), median (IQR) & $0.6(0.3,1.0)$ & $0.8(0.5,1.3)$ & 0.09 \\
\hline $\operatorname{lgG}(\mathrm{g} / \mathrm{l})$, median (IQR) & $14.1(10.3,17.3)$ & $13.1(8.9,17.9)$ & 0.77 \\
\hline ESR (mm/h),median (IQR) & $56.0(40,66)$ & $27.0(13,58)$ & $0.01^{*}$ \\
\hline CRP (mg/l), median (IQR) & $11.7(5.0,37.0)$ & $2.9(0.9,14.3)$ & $0.002^{*}$ \\
\hline \multicolumn{4}{|l|}{ Treatments prior to IM } \\
\hline $\begin{array}{l}\text { Median-to-high dose of } \\
\text { GC, } n(\%)\end{array}$ & $11(34.4)$ & $6(9.4)$ & $<0.001^{*}$ \\
\hline CYC, $n(\%)$ & $9(28.1)$ & $8(12.5)$ & 0.06 \\
\hline MMF, $n(\%)$ & $3(9.4)$ & $8(12.5)$ & 0.65 \\
\hline CsA, $n(\%)$ & $3(9.4)$ & $8(12.5)$ & 0.65 \\
\hline MTX, n (\%) & $2(6.3)$ & $6(9.4)$ & 0.60 \\
\hline
\end{tabular}

CRP C-reactive protein, CSA cyclosporine A, CTD connective tissue disease, CYC cyclophosphamide, ESR erythrocyte sedimentation rate, GC glucocorticoid, IM invasive mycoses, IQR interquartile range, MMF mycophenolate mofetil, MTX methotrexate, SD standard deviation

*Significant

and median-to-high dose of GC were included into the logistic regression analysis. Results of the logistic regression analysis are presented in Table 5. Lymphopenia $(\mathrm{OR}=3.28,95 \% \quad \mathrm{CI} 1.29-8.38, \quad P=0.01)$ and median-to-high dose of $\mathrm{GC}(\mathrm{OR}=3.40$, 95\% CI $1.04-$ 11.13, $P=0.04$ ) were associated with IM in patients with CTD.

\section{Subgroup analysis in patients with or without lymphopenia}

Patients with CTD were divided into two groups according to the lymphocyte count. A subgroup analysis showed that patients with lymphopenia were more likely to develop co-infection than those without (50.0\% vs $0 \%, P=0.01)$. Infection-specific mortality was remarkably high $(45.5 \%$ vs $0 \%, P=0.01)$ in patients with lymphopenia (Table 6). 
Table 5 Factors associated with IM in patients with CTD

\begin{tabular}{|c|c|c|c|c|c|c|}
\hline \multirow[t]{2}{*}{ Characteristics } & \multicolumn{3}{|c|}{ Univariate logistic regression } & \multicolumn{3}{|c|}{ Multivariate logistic regression } \\
\hline & Crude OR & $95 \% \mathrm{Cl}$ & $P$ value & Adjusted OR & $95 \% \mathrm{Cl}$ & $P$ value \\
\hline Interstitial pneumonia & 5.74 & $1.05-31.46$ & $0.04^{*}$ & - & - & - \\
\hline Lymphopenia & 3.50 & $1.40-8.76$ & $0.01^{*}$ & 3.28 & $1.29-8.38$ & $0.01^{*}$ \\
\hline Median-to-high dose of GC & 3.78 & $1.21-11.83$ & $0.02^{*}$ & 3.40 & $1.04-11.13$ & $0.04^{*}$ \\
\hline
\end{tabular}

Cl confidence interval, CTD connective tissue disease, GC glucocorticoid, IM invasive mycoses, OR odds ratio

"Significant

\section{Discussion}

In the present study, we found that patients with CTD were predisposed to IM. Patients with lymphopenia or treated with median-to-high dose of GC experienced high risk of IM and need fast and effective methods such as sputum culture or GM test in BALF to unveil the diagnosis. Patients with lymphopenia and impaired kidney function were extremely risky for fatal IM. Close monitoring is necessary in susceptible population to distinguish IM.

In our research, the incidence of IM was the highest in AAV (1.5\%), compared to $0.5 \%$ in the SLE population [22]. Charles et al. reviewed 80 patients with AAV treated with rituximab and found three cases of aspergillosis (3.8\%) [23]. Yoo et al. retrospectively reviewed 154 AAV patients and found 6 of them (3.9\%) were infected with Aspergillus spp. [24]. Previous reports and ours suggested that patients with AAV were susceptible to IM. Therefore, clinicians should remain vigilant for IM, especially in AAV patients. Further research exploring the characteristics and determinants of fungal infection in AAV patients is necessary.
The lung is demonstrated to be the primary site of infection, with hidden sites including the sphenoid sinus. A prospective study on transplant recipients showed that most of the aspergillosis (78\%) and zygomycosis (56\%) was limited to the lung, and other infective sites also included the sinus (13\%), cutaneous (13\%) and disseminated sites (9\%) [25]. Our research suggested that IM could occur in atypical sites in immunocompromised hosts, and careful examination is needed to uncover the potential fungal infection.

Although pathological evidence is the gold standard in diagnosing IM, the complex procedure usually delays prompt treatment. Alternatively, mycological culture and indirect test including the G test and GM test are recommended as valuable methods to facilitate IM diagnosis in immunocompromised hosts [19]. In our research, the positive rate of rapid diagnostic tests for IM was approximately $70.0 \%$. Consistent with our results, GM antigen in BALF was suggested as a promising indicator in the diagnosis of mold infection [26, 27]. Our results further supported that sputum culture and GM test in BALF were rapid diagnostic methods for IM in patients with CTD.

Table 6 Comparison between patients with or without lymphopenia

\begin{tabular}{|c|c|c|c|}
\hline Characteristics & Normal $(n=10)$ & Lymphopenia $(n=22)$ & $P$ value \\
\hline \multicolumn{4}{|l|}{ Demographic characteristics } \\
\hline Interstitial pneumonia, n (\%) & $1(10.0)$ & $5(22.7)$ & 0.39 \\
\hline Nephritis, $n$ (\%) & $4(40.0)$ & $15(68.2)$ & 0.13 \\
\hline Bilateral lung involvement, $n$ (\%) & $4(40.0)$ & 15(68.2) & 0.13 \\
\hline Co-infection, $n(\%)$ & $0(0)$ & $11(50.0)$ & $0.01^{*}$ \\
\hline \multicolumn{4}{|l|}{ Laboratory data } \\
\hline Anemia, $n(\%)$ & $5(50.0)$ & $15(68.2)$ & 0.32 \\
\hline Hypoalbuminemia, $n$ (\%) & $5(50.0)$ & 18(81.8) & 0.06 \\
\hline $\operatorname{lgM}(\mathrm{g} / \mathrm{l})$, median (IQR) & $0.9(0.4,1.1)$ & $0.5(0.3,0.9)$ & 0.24 \\
\hline $\operatorname{lgG}(\mathrm{g} / \mathrm{l})$, median (IQR) & $16.1(8.6,17.8)$ & $14.1(12.1,16.7)$ & 0.83 \\
\hline \multicolumn{4}{|l|}{ Treatments prior to IM } \\
\hline Median-to-high dose of GC, $n(\%)$ & $2(20.0)$ & $9(40.9)$ & 0.25 \\
\hline Use of immunosuppressant, $n(\%)$ & $3(30.0)$ & $12(54.5)$ & 0.20 \\
\hline Mortality, $n(\%)$ & $0(0)$ & $10(45.5)$ & $0.01^{*}$ \\
\hline
\end{tabular}

GC glucocorticoid, IQR interquartile range *Significant 
High mortality of mycoses is widely recognized. One third of the patients (31.3\%) with CTD died in our research. P. marneffei was the most fatal. These patients shared common clinical features of leukopenia, lymphopenia, and kidney impairment. Due to severe immunodeficiency, patients tended to develop co-infection with various pathogens. Practitioners may be able to influence the prognosis of IM if they remain mindful of the associated factors mentioned above and the increased risk of co-infection.

The toxicity of GC is well-recognized in immunocompromised hosts. GC use is also known to increase the risk of mix mold pulmonary infection in patients with hematological malignancies [28]. A high dose of GC was associated with the highest rate of serious infection in lupus patients [29]. Consistent with that, a median-to-high dose of GC was associated with IM and increased the risk 3.4-fold in our research. Lymphopenia was also significantly predominant in patients with IM than in those without. A cohort research has revealed that the risk of infection increased in parallel with the severity of lymphopenia in AAV patients [30]. The above findings suggested that lymphocyte count was a strong indicator for immunity surveillance. Accordingly, a subgroup analysis in the present study revealed that patients with lymphopenia were likely to develop mix infection and were associated with higher rates of mortality, and thus clinical vigilance is crucial to monitor the lymphocyte count during immunosuppressive treatment.

Our study has some drawbacks. The data used in this study was generated from one tertiary hospital from China. Thus, it could limit the generalizability of our findings to other populations. Furthermore, since this is a single-center research, only a small number of patients fulfilled the inclusion criteria. Conducting a larger study would strengthen and validate our result on the identified factors associated with IM. Additionally, biological agents are an important medication to treat CTD. However, no patient was given biologics prior to enrollment in our study. Biologics are mainly prescribed to patients with RA or SpA in China, but only a small number of patients with RA were included in this study. The impact of biologics on IM needs to be addressed in further research.

\section{Conclusions}

This study demonstrated that IM are common comorbidities in patients with CTD, especially with AAV. Sputum culture and GM test in BALF, as fast and effective diagnostic methods, should be performed in suspected patients. Patients would benefit from close monitoring of the lymphocyte count, kidney function, and co-infection to reduce mortality.

\section{Appendix}

Table 7 The International Classification of Diseases-10 coding of CTD and invasive mycoses

\begin{tabular}{|c|c|}
\hline Disease & ICD-10 coding \\
\hline \multicolumn{2}{|l|}{ CTD } \\
\hline MCTD & M35.105 \\
\hline PM/DM & $\begin{array}{l}\text { M60.992, M60.896, M33.905, M33.202+, M33.201, } \\
\text { M33.102+, M33.101 }\end{array}$ \\
\hline pSS & M35.001 \\
\hline RA & $\begin{array}{l}\text { M06.991, M06.881, M06.001, M05.951, M05.309+, } \\
\text { M05.306+, M05.303+, M05.302, M05.301, M05.001 }\end{array}$ \\
\hline SLE & M32.901, M32.155+ \\
\hline SpA & M45.X91, L40.501+ \\
\hline SSC & M34.902, M34.901, M34.807+, M34.806+, M34.801+ \\
\hline Vasculitis & $\begin{array}{l}\text { D89.153, D69.001, N05.903, M31.003, M30.101, } \\
\text { M30.001, L95.902, L95.901, 177.851, I77.607, } \\
\text { I77.605, I77.502, I77.601, M31.601, M31.505 }\end{array}$ \\
\hline \multicolumn{2}{|l|}{ Invasive mycoses } \\
\hline Aspergillosis & $\begin{array}{l}\text { B44.051+, B44.101+, B44.102+, B44.103+, } \\
\text { B44.151, B44.751, B44.752, B44.801, B44.901 }\end{array}$ \\
\hline Coccidioidomycosis & $\begin{array}{l}\text { B38.052+, B38.151+, B38.201+, B38.451+, } \\
\text { B38.751, B38.752, B38.901 }\end{array}$ \\
\hline Fungal disease & B49.X51 \\
\hline Mucormycosis & $\begin{array}{l}\text { B46.001+, B46.151+, B46.251+, B46.451, B46.452, } \\
\text { B46.501 }\end{array}$ \\
\hline Mycosis & $\begin{array}{l}\text { B48.751, B49.XO1, B49.XO2, B49.XO4+, B49.XO5, } \\
\text { B49.XO6, B49.XO7+, B49.XO9+, B49.X10+, } \\
\text { B49.X11, B49.X12+, B49.X13+, B49.X14+, B49.X15, } \\
\text { B49.X16+ }\end{array}$ \\
\hline Penicilliosis & B48.451 \\
\hline Zygomycosis & B46.952 \\
\hline
\end{tabular}

ICD-10 International Classification of Diseases-10, CTD connective tissue disease, MCTD mixed connective tissue disease, $P M / D M$ polymyositis/dermatomyositis, $p S S$ primary Sjogren's syndrome, RA rheumatoid arthritis, SLE systemic lupus erythematosus, SpA spondyloarthrosis, SSC systemic sclerosis

\section{Additional files}

Additional file 1: Figure S1. Radiographic and pathological manifestation of invasive mycoses (IM) in patients with connective tissue disease (CTD). (A) Computed tomography (CT) scan revealed soft tissue density (red arrow) filled in the sphenoid sinus. (B) T1-weighted magnetic resonance imaging (MRI) showed mildly increased signal intensity content (red arrow) in the sphenoid sinus. (C) Opacity with hypointensity (red arrow) was showed on the T2-weighted MRI. (D) Branched, septate hyphae of Aspergillus spp. (asterisks) were found in nasal sinus tissue obtained from nasal endoscopic biopsy (hematoxylin and eosin, HE staining, $\times 400$ ). (E) A mass with blurred edge (red arrow) was located in the right lung. (F) An intracavitary nodule (red arrow) was shown on a background of pulmonary bullae in the left lung. (G) Fungal hyphae (red arrows) were observed in lung tissue obtained from bronchofibroscopy (HE staining, $\times 400)$. (H) Septate fungal hyphae (red arrows) were stained brown by periodic acid-silver methenamine (PASM) in lung tissue $(\times 400)$. (I) Periodic Acid-Schiff stain (PAS) revealed fungal hyphae (red arrows) in the lung tissue $(\times 400)$. (TIF $13028 \mathrm{~kb})$ 
Additional file 2: Table S1. Distributions of the infective sites and COinfective agents in patients with CTD. CTD, connective tissue disease; MRSA, methicillin-resistant Staphylococcus aureus. (DOCX 22 kb)

\section{Abbreviations}

AAV: ANCA-associated vasculitis; BALF: Bronchoalveolar lavage fluid; Cl: Confidence interval; CRP: C-reactive protein; CSA: Cyclosporine A $\mathrm{CT}$ : Computed tomography; CTD: Connective tissue disease: CYC: Cyclophosphamide; ESR: Erythrocyte sedimentation rate; GC: Glucocorticoid; GM: Serum galactomannan; HE: Hematoxylin and eosin; ICU: Intensive care unit; IFD: Invasive fungal disease; IPA: Invasive pulmonary aspergillosis; MCTD: Mixed connective tissue disease; MMF: Mycophenolate mofetil; MRI: Magnetic resonance imaging; MTX: Methotrexate; OR: Odds ratio; PAS: Periodic Acid-Schiff stain; PASM: Periodic acid-silver methenamine; PM/DM: Polymyositis/dermatomyositis; RA: Rheumatoid arthritis; SLE: Systemic lupus erythematosus; SpA: Spondyloarthrosis; SSc: Systemic sclerosis

\section{Acknowledgements}

Not applicable.

\section{Funding}

This project was supported by grants from the National Natural Science Foundation of China $(81601403,81603435)$ and grants from the Natural Science Foundation of Guangdong Province (2018A0303130294)

\section{Availability of data and materials}

Data sharing is not applicable to this article as no datasets were generated or analyzed during the current study.

\section{Authors' contributions}

$\mathrm{ML}$ contributed to the data collection and data processing and was a majo contributor in writing the manuscript. ZZ contributed to the study design, data analysis, and manuscript preparation. FS assisted in collecting the clinical data. HL assisted in analyzing the data regarding CTD. ZY provided the pathological materials. HC contributed to the data interpretation regarding IFD. LL contributed to the critical revision of the manuscript. DC contributed to the study design, data processing, and critical revision of the manuscript. All authors read and approved the final manuscript.

\section{Ethics approval and consent to participate}

Ethics committee of the First Affiliated Hospital of Sun Yat-sen University approved the research. This work was conducted in compliance with the Declaration of Helsinki principles.

\section{Consent for publication}

Not applicable.

\section{Competing interests}

The authors declare that they have no competing interests.

\section{Publisher's Note}

Springer Nature remains neutral with regard to jurisdictional claims in published maps and institutional affiliations.

\section{Author details}

'Department of Rheumatology \& Geriatrics, The First Affiliated Hospital of Sun Yat-sen University, Guangzhou, China. ²Department of Rheumatology, The First Affiliated Hospital of Sun Yat-sen University, 58 Zhongshan 2nd Road, Guangzhou 510080, China. ${ }^{3}$ Department of Pathology, The Seventh Affiliated Hospital of Sun Yat-sen University, Shenzhen, China. ${ }^{4}$ Department of Respirology, The First Affiliated Hospital of Sun Yat-sen University, Guangzhou, China.
Received: 6 November 2018 Accepted: 20 February 2019

Published online: 11 March 2019

\section{References}

1. Goldblatt F, Chambers S, Rahman A, Isenberg DA. Serious infections in British patients with systemic lupus erythematosus: hospitalisations and mortality. Lupus. 2009:18:682-9.

2. Wu XY, Yang M, Xie YS, Xiao WG, Lin J, Zhou B, Guan X, Luo CN, Che N, Liu $X Z$, et al. Causes of death in hospitalized patients with systemic lupus erythematosus: a 10-year multicenter nationwide Chinese cohort. Clin Rheumatol. 2019:38:107-115.

3. Yang L, Xie H, Liu Z, Chen Y, Wang J, Zhang H, Ge Y, Hu W. Risk factors for infectious complications of ANCA-associated vasculitis: a cohort study. BMC Nephrol. 2018;19:138.

4. Wangkaew S, Prasertwitayakij N, Phrommintikul A, Puntana S, Euathrongchit J. Causes of death, survival and risk factors of mortality in Thai patients with early systemic sclerosis: inception cohort study. Rheumatol Int. 2017;37: 2087-94.

5. Murray SG, Schmajuk G, Trupin L, Lawson E, Cascino M, Barton J, Margaretten M, Katz PP, Yelin EH, Yazdany J. Infection is the leading cause of hospital mortality in patients with dermatomyositis/ polymyositis: data from a population based study. Arthritis Care Res (Hoboken). 2015;67:673-80.

6. Slavin MA, Chakrabarti A. Opportunistic fungal infections in the Asia-Pacific region. Med Mycol. 2012;50:18-25.

7. Sahin SZ, Akalin H, Ersoy A, Yildiz A, Ocakoglu G, Cetinoglu ED, Dizdar OS, Kazak E, Ener B. Invasive Fungal Infections in Renal Transplant Recipients: Epidemiology and risk factors. Mycopathologia. 2015;180:43-50.

8. Wang LR, Barber CE, Johnsona S, Barnabe C. Invasive fungal disease in systemic lupus erythematosus: a systematic review of disease characteristics, risk factors, and prognosis. Semin Arthritis Rheum. 2014;44:325-30.

9. Martínez-Martínez MU, Herrera-Van Oosdam D, Roman-Acosta S, Magaña-Aquino M, Baranda-Cándido L, Abud-Mendoza C. Invasive fungal infections in patients with systemic lupus erythomatosus. J Rheumatol. 2012:39:1814-8.

10. Santamaría-Alza Y, Sánchez-Bautista J, Fajardo-Rivero JF, Figueroa CL. Invasive fungal infections in Colombian patients with systemic lupus erythomatosus. Lupus. 2018;27:1116-22.

11. Su T, Li HC, Chen M, Gao L, Zhou FD, Wang RG, Zhang H, Li XM, Zhao MH. Invasive pulmonary aspergillosis in patients with antineutrophil cytoplasmic antibody associated vasculitis. J Clin Rheumatol. 2009;15:380-2.

12. Hidron A, Franco-Paredes C, Drenkard C. A rare opportunistic infection in a woman with systemic lupus erythematosus and multiple skin lesions. Lupus. 2009:18(12):1100-3.

13. Hochberg MC. Updating the American College of Rheumatology revised criteria for the classification of systemic lupus erythomatosus. Arthritis Rheum. 1997;40:1725.

14. Arnett FC, Edworthy SM, Bloch DA, McShane DJ, Fries JF, Cooper NS, Healey LA, Kaplan SR, Liang MH, Luthra HS. The American rheumatism association 1987 revised criteria for the classification of rheumatoid arthritis. Arthritis Rheum. 1988:31(3):315-24

15. Jennette JC, Falk RJ, Andrassy K, Bacon PA, Churg J, Gross WL, Hagen EC, Hoffman GS, Hunder GG, Kallenberg CG, et al. Nomenclature of systemic vasculitides. Proposal of an international consensus conference. Arthritis Rheum 1994:37:187-92.

16. Jennette JC, Falk RJ, Bacon PA, Basu N, Cid MC, Ferrario F, Flores-Suarez LF, Gross WL, Guillevin L, Hagen EC, et al. 2012 revised International Chapel Hill Consensus Conference Nomenclature of Vasculitides. Arthritis Rheum. 2013; 65:1-11.

17. Bohan A, Peter JB. Polymyositis and dermatomyositis (first of two parts). N Engl J Med. 1975;292:344-7.

18. American Rheumatism Association. Preliminary criteria for the classification of systemic sclerosis (scleroderma). Subcommittee for scleroderma criteria of the American rheumatism association diagnostic and therapeutic criteria committee. Arthritis Rheum. 1980;23:581-90

19. De Pauw B, Walsh TJ, Donnelly JP, Stevens DA, Edwards JE, Calandra T, Pappas PG, Maertens J, Lortholary O, Kauffman CA, et al. Revised definitions of invasive fungal disease from the European Organization for Research and Treatment of Cancer/Invasive Fungal Infections Cooperative Group and the National Institute of Allergy and Infectious Diseases Mycoses. Clin Infect Dis. 2008;46:1813-21. 
20. Chen D, Xie J, Chen H, Yang Y, Zhan Z, Liang L, Yang X. Infection in Southern Chinese Patients with Systemic Lupus Erythematosus: Spectrum drug resistance, outcomes, and risk factors. J Rheumatol. 2016;43:1650-66.

21. Group of Pulmonary Vascular and Interstitial Diseases Associated with Rheumatic Diseases, Chinese Association of Rheumatology and Immunology Physicians, Chinese Rheumatic Disease Data Center. 2018 Chinese expert-based consensus statement regarding the diagnosis and treatment of interstitial lung disease associated with connective tissue diseases. Zhonghua Nei Ke Za Zhi. 2018;57:558-65.

22. Lao M, Wang X, Ding M, Yang Z, Chen H, Liang L, Zhan Z, Chen D. Invasive fungal disease in patients with systemic lupus erythematosus from Southern China: a retrospective study. Lupus. 2019;28(1):77-85.

23. Charles P, Néel A, Tieulié N, Hot A, Pugnet G, Decaux O, Marie I, Khellaf M Kahn JE, Karras A, et al. Rituximab for induction and maintenance treatment of ANCA-associated vasculitides: a multicentre retrospective study on 80 patients. Rheumatology. 2014;53:532-9.

24. Yoo J, Jung SM, Song JJ, Park YB, Lee SW. Birmingham vasculitis activity and chest manifestation at diagnosis can predict hospitalised infection in ANCAassociated vasculitis. Clin Rheumatol. 2018;37:2133-41.

25. Pappas PG, Alexander BD, Andes DR, Hadley S, Kauffman CA, Freifeld A, Anaissie EJ, Brumble LM, Herwaldt L, Ito J, et al. Invasive fungal infections among organ transplant recipients: results of the transplant-associated infection surveillance network (TRANSNET). Clin Infect Dis. 2010;50:1101-11.

26. Guo YL, Chen YQ, Wang K, Qin SM, Wu C, Kong JL. Accuracy of BAL galactomannan in diagnosing invasive aspergillosis: a bivariate metaanalysis and systematic review. Chest. 2010;138:817-24.

27. Tabarsi P, Soraghi A, Marjani M, Zandian P, Baghaei P, Najafizadeh K, Droudinia A, Sarrafzadeh SA, Javanmard P, Mansouri D. Comparison of serum and bronchoalveolar lavage galactomannan in diagnosing invasive aspergillosis in solid organ transplant recipients. Exp Clin Transpl. 2012;10:278-81.

28. Magira EE, Jiang Y, Economides M, Tarrand J, Kontoyiannis DP. Mixed mold pulmonary infections in haematological cancer patients in a tertiary care cancer centre. Mycoses. 2018;61:861-7.

29. Singh JA, Hossain A, Kotb A, Wells G. Risk of serious infections with immunosuppressive drugs and glucocorticoids for lupus nephritis: a systematic review and network meta-analysis. BMC Med. 2016;14:137.

30. Goupil R, Brachemi S, Nadeau-Fredette AC, Déziel C, Troyanov Y, Lavergne $\checkmark$, Troyanov S. Lymphopenia and treatment-related infectious complications in ANCA-associated Vasculitis. Clin J Am Soc Nephrol. 2013;8:416-23.

Ready to submit your research? Choose BMC and benefit from:

- fast, convenient online submission

- thorough peer review by experienced researchers in your field

- rapid publication on acceptance

- support for research data, including large and complex data types

- gold Open Access which fosters wider collaboration and increased citations

- maximum visibility for your research: over $100 \mathrm{M}$ website views per year

At $\mathrm{BMC}$, research is always in progress.

Learn more biomedcentral.com/submissions 